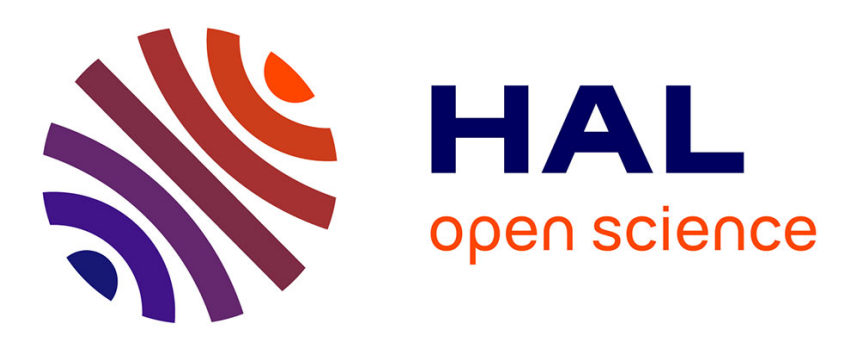

\title{
Atmospheric microwave-induced plasmas in Ar/H2 mixtures studied with a combination of passive and active spectroscopic methods
}

\author{
J M Palomares, E I Iordanova, A Gamero, A Sola, M V D Mullen
}

\section{- To cite this version:}

J M Palomares, E I Iordanova, A Gamero, A Sola, M V D Mullen. Atmospheric microwave-induced plasmas in $\mathrm{Ar} / \mathrm{H} 2$ mixtures studied with a combination of passive and active spectroscopic methods. Journal of Physics D: Applied Physics, 2010, 43 (39), pp.395202. 10.1088/0022-3727/43/39/395202 . hal-00569717

\section{HAL Id: hal-00569717 https://hal.science/hal-00569717}

Submitted on 25 Feb 2011

HAL is a multi-disciplinary open access archive for the deposit and dissemination of scientific research documents, whether they are published or not. The documents may come from teaching and research institutions in France or abroad, or from public or private research centers.
L'archive ouverte pluridisciplinaire HAL, est destinée au dépôt et à la diffusion de documents scientifiques de niveau recherche, publiés ou non, émanant des établissements d'enseignement et de recherche français ou étrangers, des laboratoires publics ou privés. 


\title{
Atmospheric microwave induced plasmas in $\mathrm{Ar} / \mathrm{H}_{2}$ mixtures studied with a combination of passive and active spectroscopic methods
}

\author{
J M Palomares $^{1 * *}$, E I Iordanova ${ }^{2}$, A Gamero ${ }^{1 *}$, A Sola ${ }^{1}$ and J J A M v d Mullen ${ }^{1,2}$ \\ ${ }^{1}$ Departamento de Física, Universidad de Còrdoba, Campus de Rabanales; ed. C-2, \\ 14071 Còrdoba, Spain \\ 2 Department of Applied Physics, Eindhoven University of Technology, PO Box 513, \\ 5600 MB Eindhoven, The Netherlands
}

E-mail: $\underline{\text { fa1garoa@uco.es }}{ }^{*}$ and f02palij@ gmail.com ${ }^{* *}$

\begin{abstract}
Several active and passive diagnostic methods have been used to study atmospheric microwave induced plasmas created by a surfatron operating at a frequency of $2.45 \mathrm{GHz}$ and with power values between 57 and $88 \mathrm{~W}$. By comparing the results with each other, insight is obtained into essential plasma quantities, their radial distributions and the reliability of the diagnostic methods. Two laser techniques have been used, namely Thomson scattering (TS) for the determination of the electron density, $n_{e}$, and temperature, $T_{e}$, and Rayleigh scattering (RyS) for the determination of the heavy particle temperature, $T_{g}$. In combination, three passive spectroscopic techniques are applied, the line broadening of the $\mathrm{H}_{\beta}$ line to determine $n_{e}$, and two methods of absolute intensity measurements to obtain $n_{e}$ and $T_{e}$. The active techniques provide spatial resolution in small plasmas with sizes in the order of $0.5 \mathrm{~mm}$. The results of $n_{e}$ measured with three different methods show good agreement, independent of the plasma settings. The $T_{e}$ values obtained with two techniques are in good agreement for the condition of a pure argon plasma, but they show deviations when $\mathrm{H}_{2}$ is introduced. The introduction of a small amount $(0.3 \%)$ of $\mathrm{H}_{2}$ into an argon plasma induces contraction, reduces $n_{e}$, increases $T_{e}$, enhances the departure from equilibrium and leads to conditions that are close to those found in cool atmospheric plasmas.
\end{abstract}

PACS: $52.70-\mathrm{m} ; 52.70 . \mathrm{kz} ; 52.25 .0 \mathrm{~s}$

Keywords: cool atmospheric plasmas, surfatron discharges, Thomson scattering, Rayleigh scattering, absolute intensity measurements, Stark broadening.

\section{Introduction}

Gas discharges working in the open air are nowadays subjects of growing interest. This is due to their applicability in many technological fields such as material processing, waste treatment, spectrochemical analysis, surface treatment and biomedical applications. But the most important reason behind their popularity is that they are relatively easy to operate. One consequence of the 
operation in open air is that this might create and/or enhance deviations from equilibrium. For example large differences between the gas temperature $T_{g}$ and the electron temperature $T_{e}$ can be expected when the power density and the (radial) size of the plasma are small. The reason is that in small-sized plasmas the heavy particles are effectively cooled by the environment whereas the electrons are heated by the EM field. This, together with the fact that the small ratio of the mass of the electron and the heavy particles obstructs the heat transfer between the two groups, may lead to large $T_{e} / T_{g}$ values.

This especially holds for the class of Cool Atmospheric Plasmas (CAPs) for which the temperature ratio can reach values up to $T_{e} / T_{g}=50$. The low gas temperature of $330 \mathrm{~K}$ or lower prevents the plasma-treated material to be destroyed by heat overloading; the plasma-material interaction is determined by the exchange of radicals rather than by heat transfer. Examples of CAPs are the micro plasmas [1], the plasma needle [2] and the plasma pencil [3].

The characterization of a CAP is very difficult. Since it is small in volume and often filamentary in shape the internal structure can not be investigated easily. Moreover, CAPs are strongly influenced by the environment. Air molecules can penetrate and mix with atoms or ions of the main gas, e.g. Ar. This leads to molecular channels that offer effective recombination paths. Consequences of this molecular assisted recombination are that the electron density will go down whereas the electron temperature increases. So apart from the thermal non-equilibrium feature many other plasma aspects will be brought out of equilibrium as well. It is expected that the atomic state distribution (ASDF) will strongly deviate from the shape as prescribed by Boltzmann's law while the ionization degree will not obey the Saha formula. This makes the characterization of these plasmas far from easy. The larger the deviation from equilibrium, the less we can rely upon the classical laws of statistical physics and the more the various plasma aspects are decoupled from each other. So, in order to get a valuable characterization much more parameters have to be specified. Moreover, due to the equilibrium departure, we can no longer blindly rely upon the Optical Emission Spectrometry (OES); especially not if the methods are based on distribution laws of equilibrium statistical mechanics, such as in the case of the Boltzmann-plot method.

This means that apart from OES we have to consult other methods as well; for instance those that are used in the field of laser aided diagnostics. Not only to determine the relevant plasma parameters but also to get, via a cross-validation of the different techniques, deeper insight into the mechanisms that are responsible for the departure from equilibrium. So, to get a better understanding of CAPs we have to employ the method of poly-diagnostics. An approach that was introduced in the experimental characterization of atmospheric Inductively Coupled Plasmas (ICPs) [4] and recently applied to low pressure surfatron discharges [5].

However, since the step from the robust ICP to the tiny CAPs was found to be too large it was decided to employ the poly-diagnostic approach to semi-CAPs. For this we selected atmospheric plasmas generated by means of microwaves launched by a surfatron in $\mathrm{Ar} / \mathrm{H}_{2}$ mixtures. The $T_{g}$ values of these plasmas are on the order of $1400 \mathrm{~K}$ and with values of $T_{e} / T_{g} \approx 7$ they are indeed somewhere in between thermal plasmas $\left(T_{e} / T_{g} \approx 1\right)$ and the class of CAPs. Moreover, due to their stability these surfatron induced plasmas facilitate the study of internal structures for conditions approaching those found in CAPs. Thus the idea is that, if we can characterize these semi-CAP plasmas, we can develop a strategy for future studies on the characterization of CAPs.

The plasma flame generated with the surfatron is comparable to that created with the axial injection torch (TIA) [6] and the microwave plasma torch (MPT) [7]. The main difference is that the present plasma is much more stable so that it is easier to study the radial distribution of 
plasma quantities. In order to approach CAP-conditions we reduce the power and introduce small amounts of hydrogen. While doing so we keep track of the results obtained by active and passive spectroscopy and monitor whether approaching CAP-conditions might lead to discrepancies between the methods.

Two laser diagnostic techniques have been used, namely Thomson scattering (TS) and Rayleigh scattering (RyS). TS is employed to determine the electron density $n_{e}$ and temperature $T_{e}$ with good spatial resolution [8-15]. Rayleigh scattering gives us spatially resolved heavy particle temperature $T_{g}$ [16-19]. In this way we can (among others) study and characterize the departure from equilibrium of the plasma.

In combination with the active methods, three different passive-spectroscopic techniques are applied. The electron density is obtained from the Stark broadening of the $\mathrm{H}_{\beta}$ line $[20,21]$ and the method of Absolute Continuum Intensity (ACI) measurements. The electron temperature is found by means of the Absolute Line Intensity (ALI) measurements. The methods of ACI and ALI were introduced in $[22,23]$. In [24] they were applied to the TIA.

As stated above, the conditions found in CAPs are approached by power reduction and hydrogen addition. It is found that due to these actions the plasma becomes smaller in diameter, lower in electron density and gas temperature and higher in electron temperature. With respect to the validity of the methods, the poly-diagnostic approach has shown that the $n_{e}$-values as deduced from the continuum and the Stark broadening of $\mathrm{H}_{\beta}$ are reliable. However, the electron temperature method based on the absolute line intensity measurement loses validity when hydrogen is introduced in order to approach the CAP conditions.

The paper is organized as follows: Section 2 being devoted to the experimental set-up, gives a description of the plasma source, the plasma settings and the diagnostics tools. Section 3 deals with the basic concepts of the different diagnostic methods. In section 4 the results are shown, the influence of $\mathrm{H}_{2}$ introduction and power reduction are studied and the methods are compared to each other. Finally, section 5 gives conclusions. 


\section{Experimental setup}

Figure 1 gives an overview of the setup that has been used for both passive and active spectroscopy performed on atmospheric microwave induced plasmas. It shows four sections: the atmospheric surfatron, the laser system, the detection branch for active spectroscopy and the detection branch for passive spectroscopy.

\subsection{Atmospheric surfatron setup}

Electromagnetic waves are generated in a Muegge power supply that delivers microwaves at 2.45 $\mathrm{GHz}$. The power is adjustable up to a maximum of $300 \mathrm{~W}$. Coaxial cables guide the microwaves to the surfatron launcher where they are coupled to the plasma [25]. The surfatron is equipped with two plungers that give extra tuning capabilities to achieve an optimal coupling. The microwave power absorbed by the plasma is determined by subtracting the reflected from the forward (=applied) power. The plasma is created inside a capillary quartz tube with an inner and outer diameter of $1.0 \mathrm{~mm}$ and $2.5 \mathrm{~mm}$. The electromagnetic waves propagate along the interface between plasma and tube creating and sustaining the discharge. The dielectric tube ends $1 \mathrm{~cm}$ behind the surfatron gap and the plasma expands into the open air. The measurements take place at $1 \mathrm{~mm}$ behind the end of the tube. The gas flow is set by means of mass flow controllers that allow the tuning of the flow rates and the composition of the gas mixture. The characteristics of the plasma flame mainly depend on the absorbed microwave power and the composition of the gas mixture. The dependence on the flow rate was found to be only moderate. The three plasma settings that have been investigated are listen in table 1; the flow is in all cases around $1 \mathrm{slm}$ :

Table 1. Experimental settings studied

\begin{tabular}{ccc}
\hline Condition & Absorbed power (W) & Composition \\
\hline $\mathrm{A}$ & 74 & $\mathrm{Pure} \mathrm{Ar}$ \\
$\mathrm{B}$ & 88 & $\mathrm{Ar}+0.3 \% \mathrm{H}_{2}$ \\
$\mathrm{C}$ & 57 & $\mathrm{Ar}+0.3 \% \mathrm{H}_{2}$ \\
\hline
\end{tabular}

Before the plasma settings were selected a global exploration of the plasma response to changes in the settings was made. It was found that at the position of $1 \mathrm{~mm}$ behind the tube exit, where the measurements takes place, the pure Ar plasma does not change substantially if the power and flow rate are changed moderately. A feature that was already observed in [24] for the pure argon TIA. On the other hand, the plasmas in $\mathrm{Ar}-\mathrm{H}_{2}$ mixtures are sensitive to changes in the power. This brought us to selecting the settings $\mathrm{A}, \mathrm{B}$ and $\mathrm{C}$ as given in table 1 .

\subsection{The laser}

For the frequency doubled Nd:YAG laser we used a Continuum Laser model Precision II 8010 that produces $8 \mathrm{~ns}$ pulses with a repetition rate of $10 \mathrm{~Hz}$. The pulse energy is adjustable with a maximum of $700 \mathrm{~mJ} @ 532 \mathrm{~nm}$. This makes it possible to investigate the influence of the laser power on the plasma. The guiding and focusing of the laser beam is done with a set of three mirrors and a lens with a focal length of $\mathrm{f}=1.0 \mathrm{~m}$.

\subsection{Active spectroscopy detection branch.}

The detection branch of the laser scattering techniques is formed by a set of two achromatic lenses, a triple grating spectrograph (TGS) and a cooled iCCD camera. The TGS is the same as the one described in [26, 27]. It was originally designed to reject false stray (FS) light and Rayleigh scattered (RyS) photons, and to collect and disperse the TS signal [27]. The rejection of FS and RyS is done by a combination of the first two gratings with a mask in between. This forms a notch filter. The final dispersion is performed by the third grating that sends the dispersed image to an Andor DH534 iCCD camera. For the detection of the RyS signal the same TGS is used but with the notch filter "switched off". This switch-off merely implies that the mask in 
between the first and second grating is removed. The Rayleigh photons are then collected together with the photons of FS and TS.

\subsection{Passive spectroscopy detection branch.}

The detection branch used for the OES is described in detail in [5]. The emitted plasma light is focused into an optical fiber, by which the light is guided to the entrance slit of a spectrometer. The spectrometer, a high resolution double Echelle monochromator (DEMON), consists of an Echelle spectrometer in sequence with a prism pre-monochromator. An Andor DV434 CCD is used to record the spectra.

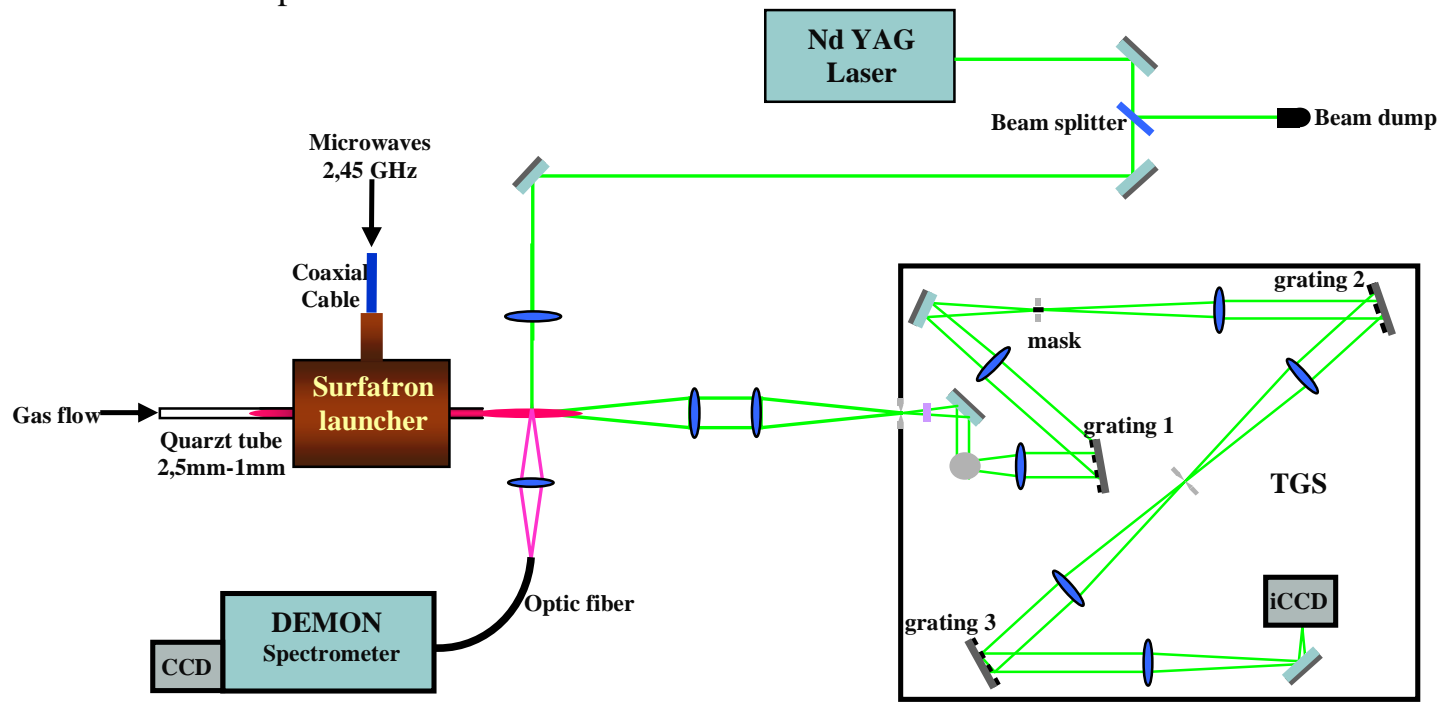

Figure 1. Schematic view of the setup showing the plasma source (atmospheric surfatron), the laser, and the detection branches for the active (TGS), and passive spectroscopy (DEMON).

\section{Spectroscopic techniques}

The spectroscopic techniques and methods used in this study have been introduced and discussed in earlier studies. Here we confine ourselves to the information that is needed to obtain a good comparison of the experimental tools with each other.

All the spectroscopic methods were applied at the same plasma position, namely at $1 \mathrm{~mm}$ behind the end of the tube. This position is chosen as being a good compromise between giving high plasma intensity, thus close enough to the tube exit, and a low FS level, that means far enough from the tube exit. The techniques were applied consecutively to the same plasma settings and employed on the same day. During the application of laser aided spectroscopy, the optical fiber was removed in order to avoid that the laser beam hits and/or damages the fiber. On the other hand, during the performance of passive spectroscopy the laser is switched-off in order to avoid any kind of reflections that could enter the fiber.

\subsection{Thomson Scattering}

The theoretical basis and experimental procedure of the TS technique have been explained in detail in previous works [12, 26, 28]. It is based on the scattering of laser light on the collection of free electrons. As such it gives direct insight in the properties of the electron gas. This is the main difference with the more indirect passive methods where the electron properties are deduced from the spectral features of the plasma. In the current setup the laser beam is aligned perpendicularly to the plasma flame axis and goes through its radial center (figure 1). The entrance of the TGS collects a scattering length of $1.2 \mathrm{~cm}[26,28]$ and since the radius of the plasma flame is smaller 
than $1 \mathrm{~mm}$, one picture taken with the TGS covers much more than the flame diameter. This makes it possible to obtain the radial profiles of $n_{e}$ and $T_{e}$ in one go.

Figure 2 gives an example of an iCCD image: in the vertical direction we find spatial information along the laser beam, while the horizontal direction of the iCCD gives spectral information for the corresponding spatial position. In the centre of figure we find the TS spectrum as created by the laser scattering on the plasma flame; below and above the TS signal we observe the Raman spectrum generated by the scattering on molecules of the air surrounding the plasma.

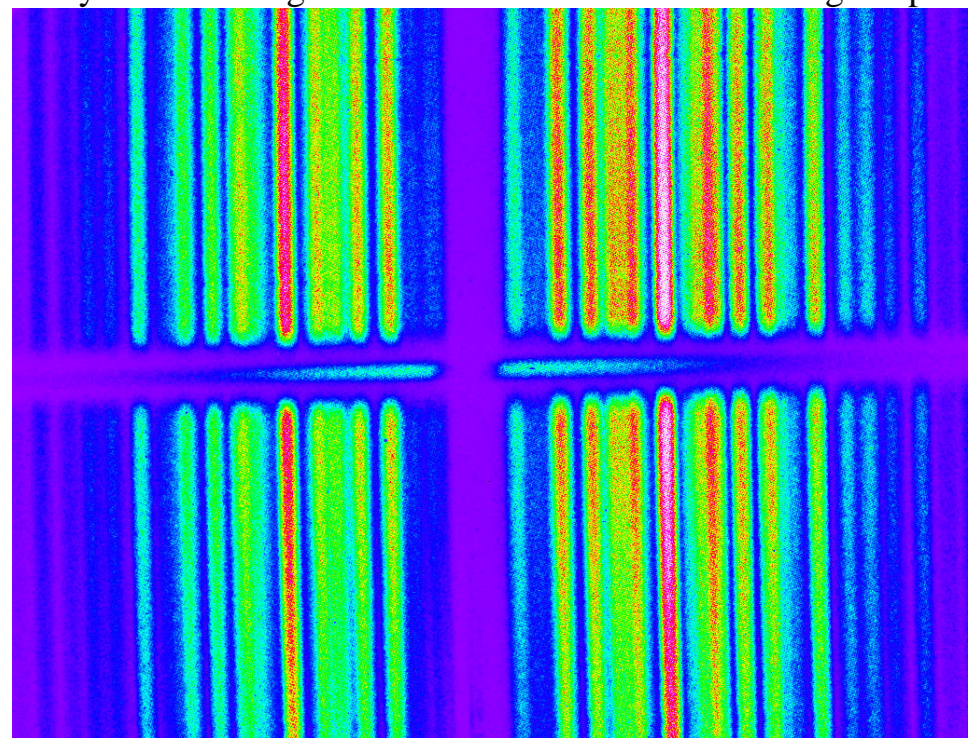

Figure 2. An iCCD image of the scattering of laser light on the plasma flame and the surrounding air. Spatial information is given in the vertical direction; spectral information in the horizontal direction. The vertical central band is the result of the filtering performed by the TGS.

Since the electron density is, as we will see, below $3.10^{21} \mathrm{~m}^{-3}$ and the electron temperature in the range $1-2 \mathrm{eV}$ we can expect that the scattering will be incoherent. This implies that the total scattered power is directly proportional to the electron density and that the TS spectrum has a Gaussian profile. The width of this profile then directly provides the electron temperature $T_{e}$ [28] while the value of $n_{e}$ can be derived from the spectral area, after an intensity calibration. As in our previous works, the calibration is achieved by measuring the Raman spectrum of nitrogen at controlled pressure [26-29].

In order to determine whether the laser power influences the plasma, different measurements were done changing the energy per pulse in a range of $40 \mathrm{~mJ}-170 \mathrm{~mJ}$. It was experimentally found that laser heating can be avoided if the pulse energy is kept below $50 \mathrm{~mJ}$. This, combined with the insights obtained in the error analysis in [28], leads to an estimation of the relative error in $n_{e}$ of $17 \%$ and $15 \%$ for $T_{e}$.

\subsection{Rayleigh Scattering}

Rayleigh Scattering (RyS) is the elastic scattering of laser photons by electrons bound to heavy particles. The method is described in [16-19] and was, among others, applied to inductively coupled plasmas, surfaguide induced plasmas and plasmas created by the TIA. Since the RyS intensity is directly proportional to the atom density while this is inversely proportional to the gas temperature, we get the plasma gas temperature by comparing the RyS power of the plasma, $P_{p l}$, with that of argon gas at room temperature, $P_{\text {room }}$. Provided the laser and observation settings are the same, the relation simply reads $T_{g}=T_{\text {room }} P_{\text {room }} / P_{p l}$. 
The Rayleigh spectra are collected with the same detection branch as that used for the TS experiments, and with the same laser alignment. However, the mask of the TGS must be removed, so that the notch filter is "switched-off". In this configuration the iCCD records three signals, the Rayleigh signal, the false stray light and the Thomson signal. The TS contribution to the total spectrum can be calculated to be less than 5\% for the plasma conditions under study. The false stray light is produced by reflections of the laser beam, or a laser side-beam, on different components of the setup close to the scattering volume. This contribution cannot be neglected and must be removed in order to derive the Rayleigh intensity. This is done by combining the measurements obtained with and without the discharge. Since the configuration is identical in both cases the FS remains unchanged, whereas the RyS signal changes according to temperature difference. The accuracy in the $T_{g}$-determination is estimated by taken the inaccuracies of the FS $(15 \%)$ and the TS contribution $(<5 \%)$ into account. The effect of a possible entrainment of air into the flame can be neglected: even assuming an excessive contamination of $10 \%$ of air $\left(\mathrm{N}_{2}\right.$ $80 \%+\mathrm{O}_{2} 20 \%$ ) leads to a relative error in the gas temperature determination smaller than $1 \%$. This because the values of the RyS cross section for argon, nitrogen and oxygen $\left(\sigma_{\mathrm{N} 2}=1.12 \sigma_{\mathrm{Ar}}\right.$, $\sigma_{\mathrm{O} 2}=0.915 \sigma_{\mathrm{Ar}}$ ) [30] are close to each other. All these considerations lead to a final estimation of the relative error for the gas temperature in the order of $15 \%$.

\subsection{Absolute intensity measurements}

We use here the passive method of Absolute Intensity Measurements (AIM) as introduced in [24] and applied to a TIA working on argon at atmospheric pressure. This method is based on the absolute measurements of the continuum and line radiation. The method of absolute continuum intensity (ACI) measurements, described in detail in [22], provides values of electron density from the continuum level for a known $T_{e}$-value. On the other hand, the method of absolute line intensity (ALI) measurements allows the construction of the atomic state distribution function (ASDF), including the ground state. From the ASDF the electron temperature can be determined, if $n_{e}$ is known, making use of a collisional radiative model (CRM) [23]. As a side product, the ASDF also provides the values of the excitation temperatures of the upper part, $T_{\text {spec }}$, and the bottom of the distribution, $T_{13}$. These three temperatures, $T_{e}, T_{13}$ and $T_{s p e c}$, give insight into the deviation from equilibrium.

In the present study the AIM method is employed in a slightly different form making use of TS results. This leads to a more precise method. The main contributions to the error of $n_{e}$ are the uncertainties in the gas temperature, $T_{g}$, and the plasma size, $D$. In the present experiment we can estimate relative uncertainties for the plasma diameter of $8 \%$ and for the gas temperature of $15 \%$. All these estimations lead to a total relative error of $26 \%$ in $n_{e}$ as obtained for the continuum and of $6 \%$ in $T_{e}$ from the line radiation.

The absolute intensity measurements are performed with the detection branch designed for the passive spectroscopy techniques. In contrast to the laser based techniques, the passive methods do not deliver spatial information. Instead they provide information on averaged values of $n_{e}$ and $T_{e}$ for the plasma cross section at a distance of $1.0 \mathrm{~mm}$ from the exit of the tube.

\subsection{Stark broadening}

To obtain the electron density $n_{e}$ from the Stark broadening of $\mathrm{H}_{\beta}$ we used the computer simulations developed by Gigosos and Cardeñosos [20, 21]. However, before this can be employed one has to correct the measured line-width for other broadening mechanisms. Estimations show that for the studied plasma conditions the resonance and natural broadenings of the $\mathrm{H}_{\beta}$ line can be neglected; that the van der Waals broadening [31] at a gas temperature of 1000 $\mathrm{K}$ gives a full-width at half-maximum $(\mathrm{FWHM})$ of $\sim 0.04 \mathrm{~nm}$, that the Doppler broadening for the 
same conditions has a FWHM of $\sim 0.01 \mathrm{~nm}$, while the monochromator has an instrumental broadening in the order of $0.005 \mathrm{~nm}$. For an electron density of $10^{21} \mathrm{~m}^{-3}$ and a gas temperature of $1000 \mathrm{~K}$ the GC model predicts a Stark width around $0.2 \mathrm{~nm}$. We may therefore conclude that the main contribution to the line width originates from Stark broadening. However, for each experimental condition all other contributions were calculated using the $T_{g}$-value as obtained by RyS; these were subtracted in order to determine the Stark width.

The measurements are carried out for each condition with the same detection branch as that used for the ACI and ALI method, and at the same position ( $1 \mathrm{~mm}$ behind the end of the tube). As in the case of ACI and ALI we do not get spatially resolved $n_{e}$-values. The experimental uncertainties in the line-width determination can be estimated to be around 7\%, what induces an error in the values of $n_{e}$ of about 13\%. The uncertainties in the gas temperature (around 15\%) induce an error in $n_{e}$ of $5 \%$. The total error bars for $n_{e}$ obtained with the Stark width has an estimated relative value of $14 \%$.

\section{Results and discussion}

As stated before, the diagnostic methods are applied on atmospheric surfatron plasmas, on the flame formed behind the end of the dielectric tube, at a distance of $1 \mathrm{~mm}$ of the tube exit. Three plasma settings were studied (table 1). In case A the plasma is generated by coupling $74 \mathrm{~W}$ to a pure argon flow. In case $\mathrm{B}$ and $\mathrm{C}$ we applied $88 \mathrm{~W}$ and $57 \mathrm{~W}$ respectively to an argon flow at which a small percentage of hydrogen $(0.3 \%)$ was admixed. By introducing hydrogen and reducing the power we intend to enhance the departure from equilibrium and to approach the conditions of cool atmospheric plasmas.

\subsection{Radial profile of the flame}

Thanks to the spatial resolution that is obtained with the laser techniques it is possible to obtain the diameter of the plasma flame and the radial profiles of the electron temperature $T_{e}$, electron density $n_{e}$ and gas temperature $T_{g}$. The diameter can directly be obtained from inspection of the iCCD images. As shown in figure 2 the iCCD camera collects spatial and spectral information. By using the known number of pixels per $\mathrm{mm}$, it is possible to measure the flame-width directly from the pictures. For the pure argon condition (case A) the plasma has a diameter of $0.66 \mathrm{~mm}$, for case B the plasma size drops to $0.44 \mathrm{~mm}$, while for case $\mathrm{C}$ the diameter is $0.25 \mathrm{~mm}$. So it is clear that the $\mathrm{H}_{2}$-introduction and power-reduction reduces the flame diameter making the influence of the environment more important. Figure 3 gives the radial profiles of $n_{e}, T_{e}$ and $T_{g}$ for the three experimental conditions mentioned above. 

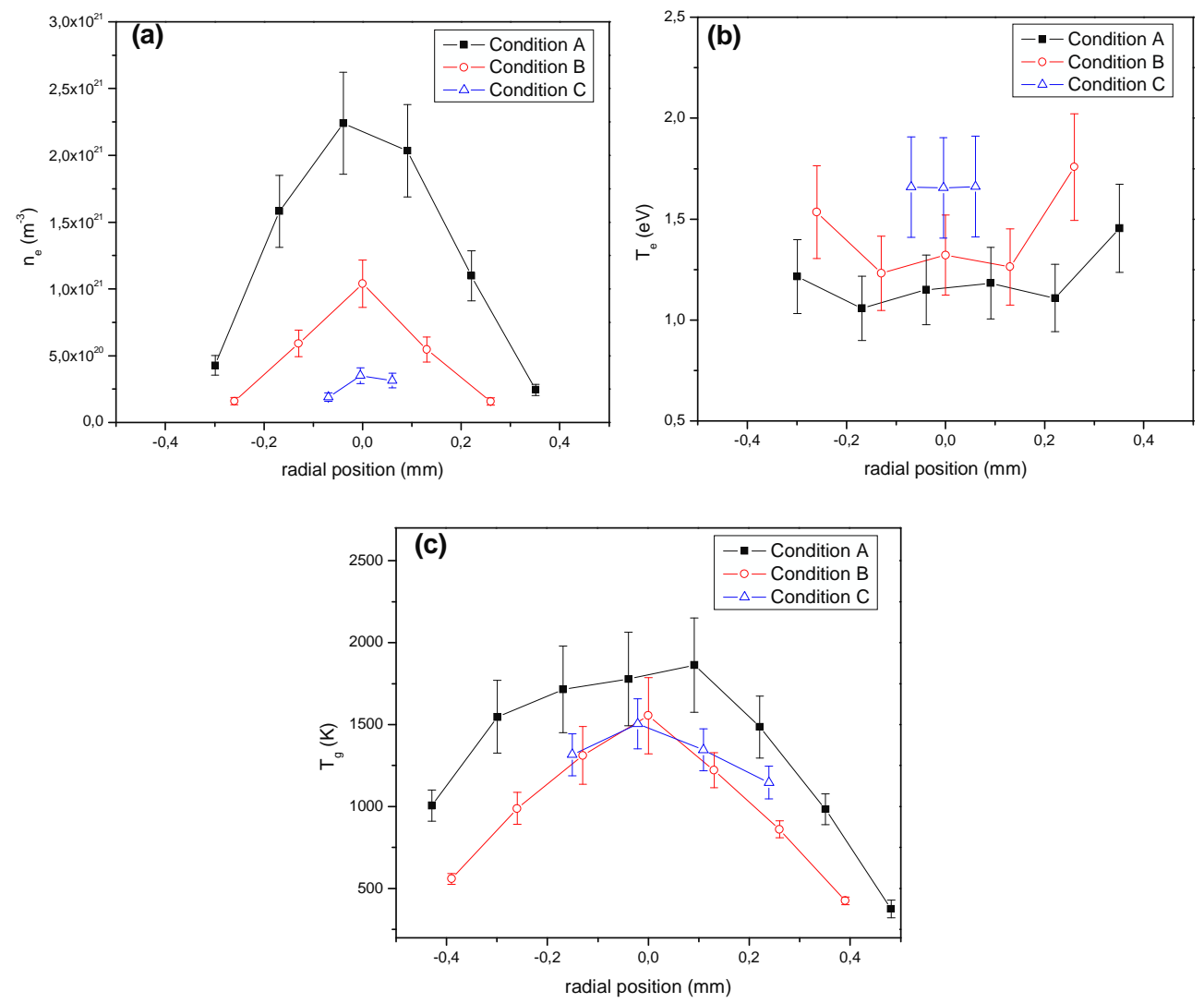

Figure 3. Radial profiles of $n_{e}$ (a), $T_{e}$ (b) and $T_{g}$ (c), for the three experimental conditions described in table 1 .

The profiles of $n_{e}$ and $T_{g}$ have similar radial shapes; there is a maximum at the center while the intensity monotonically decreases approaching the plasma edge. On the other hand, $T_{e}$ shows more or less a flat profile in the central part, with an increase approaching the edges. Thus at the flame edge, where due to merging of the plasma with the cool molecular environment the electron density drops, the electron temperature increases.

The influence of molecules at the edge of the discharge can be studied more in-depth by introducing small amount of $\mathrm{H}_{2}$ directly into the discharge. As can be seen from the figures $3 \mathrm{a}-\mathrm{c}$ this leads to radial profiles of $n_{e}$ and $T_{e}$ that are sharper, showing a stronger plasma contraction. Moreover, the change in plasma settings from A->B->C reduces internal values of $n_{e}$ while increasing $T_{e}$. It is well documented in literature the important role played by dissociative recombination processes involving the molecular ion $\mathrm{Ar}_{2}{ }^{+}$on pure atmospheric argon discharges [32-35]. In this context, the reduction of $n_{e}$ is most likely due to the fact that the introduction of hydrogen opens new loss channels for dissociative recombination of molecular ions such as $\mathrm{H}_{2}^{+}$, $\mathrm{H}_{3}{ }^{+}$, and $\mathrm{ArH}^{+}$[36-38] and that these extra recombination channels lead to a reduction of $n_{e}$. Less obvious is the increase in $T_{e}$, but this can be understood by realizing that an enhancement of the recombination has to be balanced with a higher ionization rate; this demands for an increase of $T_{e}$. Thus, there is a strong similarity between the radial change of the plasma conditions approaching the plasma edge and the global change in internal plasma parameters by introducing molecules. In both cases the decrease in $n_{e}$ (enhanced recombination) is associated with an increase in $T_{e}$ (ionization rate increase). It is instructive to compare these results with those given in [39] where the opposite route was taken: the influence of molecules from the environment on the plasma is 
studied by bringing an argon microwave induced plasma in a pure Ar environment. It was found that changing the environment from air to pure Ar increases the plasma size and the electron density whereas the electron temperature decreases. These trends, being opposite to what we found in the current study where extra molecules were added in stead of removed, support our findings that the introduction of small amounts of $\left(\mathrm{H}_{2}\right)$ molecules into an argon plasma, either by the edge or via the central flow, reduces $n_{e}$ and increases $T_{e}$.

The radial profile of $T_{g}$ has a wider shape with a smoother decay with and without impurities. This indicates that the heavy particles carry energy beyond the discharge limits. With the presence of $\mathrm{H}_{2}$ molecules the gas temperature drops. As shown by figure $3 \mathrm{c}$ the average values of $T_{\mathrm{g}}$ drops from $1550 \mathrm{~K}$ for pure argon to around $1300 \mathrm{~K}$ in the argon-hydrogen mixtures. A possible explanation is that part of the kinetic energy of the heavy particles is stored in different rotational and vibrational levels. Another factor that plays a role is that the decrease of $n_{e}$ by introducing $\mathrm{H}_{2}$ leads to a decrease of the interactions between electrons and heavy particles. This implies that less kinetic energy is transported from the electrons to heavy particles so that $T_{g}$ decreases.

\subsection{Electron density results}

The electron density has been measured with three different methods, Thomson scattering (TS), absolute continuum intensity (ACI) measurements and the Stark broadening of the $\mathrm{H}_{\beta}$ line.

Figure 4 shows the density results for the three different diagnostics. The results of $n_{e}$ obtained from Stark broadening measurements and ACI are in good agreement with those obtained by TS. To perform the comparison with the averaged results of the passive methods, the radial profiles obtained with TS in section 4.1 were averaged over the flame cross section. Apparently the admixture of hydrogen does not change the agreement between the results obtained by passive and active spectroscopy. The agreement between TS and Stark broadening was already found in the past [5]. The agreement with ACI measurements proves that this method is also reliable for the $n_{e}$-determination. However, the ACI measurements are sensitive to the values of the gas temperature, the electron temperature and the plasma size [24]. So, in order to obtain precise results the method requires good values of these parameters as well. 


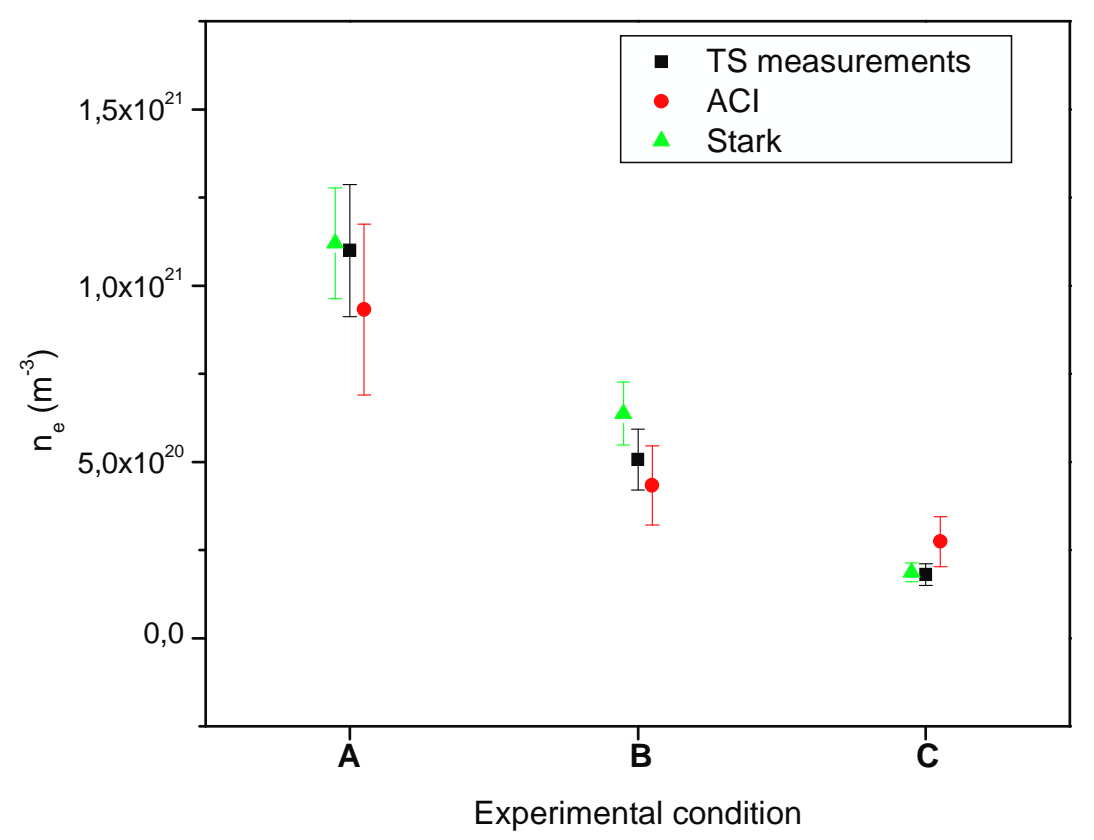

Figure 4. Electron density results obtained by the three different methods, for the three experimental conditions described in table 1 . The TS measurements (black squares) are obtained after averaging the radial profiles shown in figure 3 a over the plasma cross section.

\subsection{Electron temperature results}

The electron temperature has been measured with two methods namely TS and ALI. The ALI method is based on the bottom part of ASDF of argon [24]. By including the ASDF-top it is possible to obtain several "excitation temperatures" of the distribution. In figure 5, the argon ASDF is shown for the three experimental conditions. The first temperature, $T_{13}$, is obtained from the population ratio of the ground state and the third group of excited levels, the $4 p$ levels of argon. The second temperature, $T_{\text {spec }}$, comes from the ASDF slope as formed by the population of $4 \mathrm{p}$ and higher states. As explained before, the value of electron temperature, $T_{e}$, is obtained with the help of a collisional radiative model [24]. This transfers $T_{13}$ into $T_{e}$ taking the ionizing state of the Ar-system into account. 


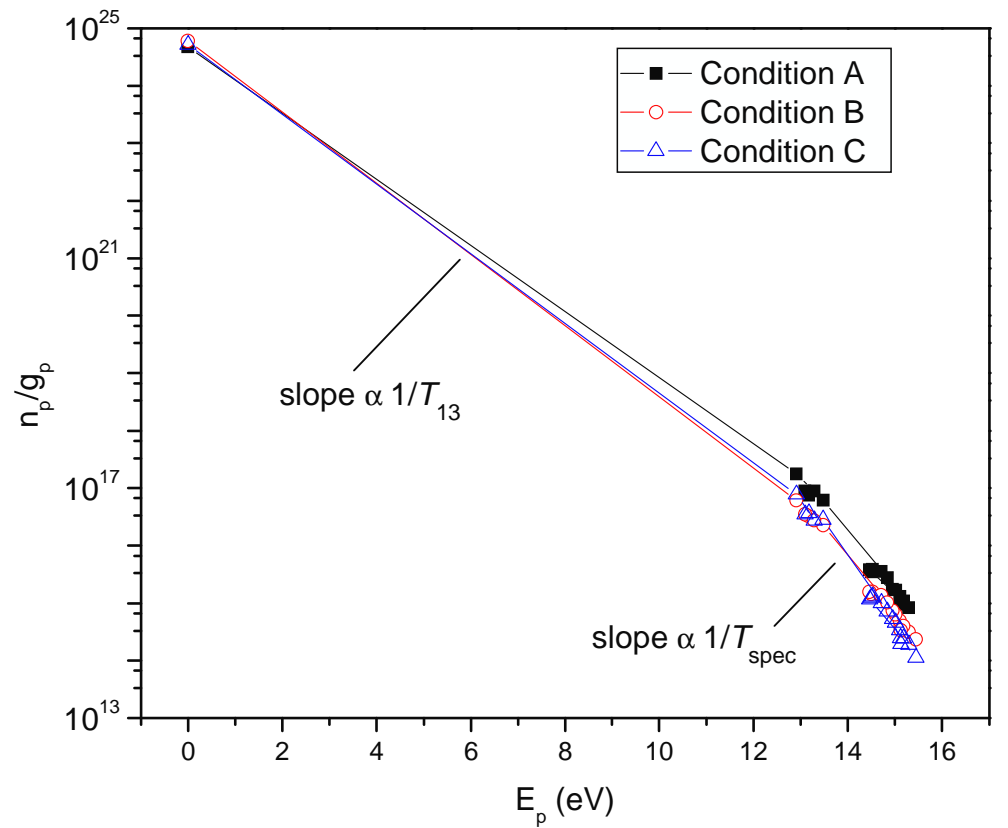

Figure 5. Argon ASDF obtained for the three experimental conditions described in table 1.

For the three experimental conditions the values obtained of $T_{13}$ and $T_{\text {spec }}$ are around $0.7 \mathrm{eV}$ and $0.45 \mathrm{eV}$ respectively. The large differences between these values clearly show that the system is strongly ionizing and thus far from equilibrium [40]. We recall that absolute intensity measurements are needed to get $T_{13}$ (and thus $T_{e}$ ). The reason is that $n_{l}$, the density of the ground state (level "1"), is not determined spectroscopically but in an absolute way via the gas law $n_{1}=$ $\mathrm{p} / \mathrm{k} T_{g}$. This implies that $n_{3}$ (the density of the level 3) must also be known absolutely. The $T_{\text {spec }}$ can be determined by ratios since all the levels are radiative. Because this is much easier to do it is seductive to confine the temperature characterization to this method. However, as shown in this study, the value obtained differs substantially from the electron temperature.

Figure 6, gives the results of the electron temperature obtained with the two techniques. As in the case of section 4.2, the radial profiles obtained with TS are averaged over the plasma cross section in order to be compared with the results obtained by ALI. The figure shows that there is a good agreement between the $T_{e}$-value obtained by TS and ALI as far as for the pure argon case (case A) concerns. The same agreement was found for the region close to the launcher of a low pressure surfatron [5]. These findings validate the ALI method for the diagnosis of the electron temperature on pure argon microwave plasmas at low and atmospheric pressure and shows that transformation $T_{13} \rightarrow T_{e}$ as done by the collisional radiative model (CRM) is successful.

However, the introduction of $\mathrm{H}_{2}$ leads to a discrepancy between the two methods. It is seen that TS indicates that $T_{e}$ rises, whereas the ALI method suggests that $T_{e}$ deceases when $\mathrm{H}_{2}$ is introduced. In section 4.1 we found as explanation for the rise in $T_{e}(\mathrm{TS})$ that the introduction of hydrogen leads to enhanced recombination and that this demands for an increase of the ionization rate and thus an increase in $T_{e}$. This reasoning supports the TS findings and suggests that the ALI method is not correct. The latter can be understood realizing that the $T_{e}$ (ALI) is obtained via $T_{13}$ and thus by the measurement of the density of the $\operatorname{Ar}(4 \mathrm{p})$ population. It is likely that the introduction of $\mathrm{H}_{2}$ changes the excitation in the argon system substantially. For instance the $\mathrm{H}_{2}$ might lead to a quenching of the $\operatorname{Ar}(4 \mathrm{p})$ levels by means of excitation transfer processes. This lowers $T_{13}$ and therefore $T_{e}$. We recall that $T_{e}$ is deduced from $T_{13}$ by making use of a CRM. 
However this CRM is based on pure Ar processes and might lose validity in $\mathrm{Ar} / \mathrm{H}_{2}$ mixtures. This explanation is supported by figure 5 which shows that the population of $4 \mathrm{p}$ drops when hydrogen is introduced.

Summarizing, we may state that the TS method, which directly probes the electron gas, gives reliable values of $T_{e}$ (and $n_{e}$ ) and is applicable for any gas mixture. However, in contrast to TS the ALI method is indirect; it derives the electron properties out of the ASDF by using a collisional radiative model. Since the CRM we used is built for a pure argon system, it is possible that it does not describe the argon ASDF in $\mathrm{Ar} / \mathrm{H}_{2}$ mixtures properly.

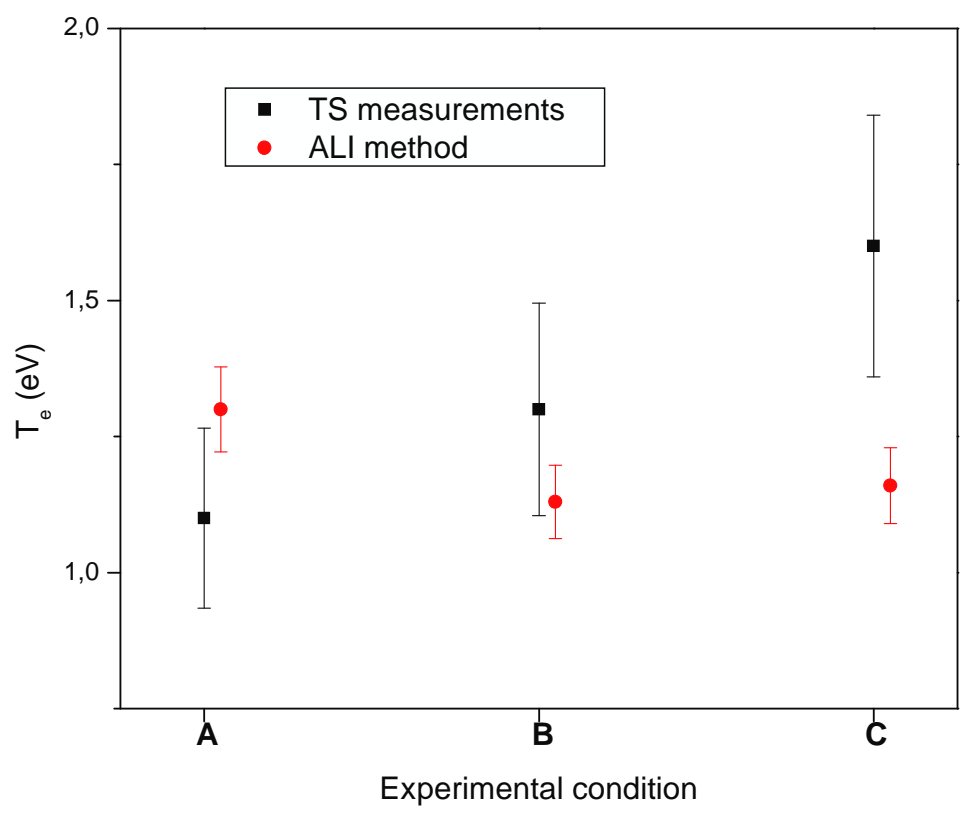

Figure 6. Electron temperature results obtained by two different techniques, for the three experimental conditions described in table 1. The TS measurements (black squares) are obtained after averaging the radial profiles shown in figure $3 \mathrm{~b}$ over the plasma cross section.

\section{Conclusions}

In this paper, different diagnostic methods and techniques have been applied to study microwave plasmas generated at atmospheric pressure. The measurements have been taken on the plasma flame produced with a surfatron, using argon as the (main) carrier gas. Due to the introduction of small amounts of hydrogen and the reduction of the microwave power it was possible to approach conditions that are typical for Cool Atmospheric Plasmas (CAP).

Two laser active techniques have been applied, Thomson and Rayleigh scattering, and three passive methods namely Stark broadening and the absolute intensity measurements of the continuum (ACI) and line emission (ALI). These methods provide values of the most important plasma parameters: the electron density and temperature, and the gas temperature.

The laser techniques have the advantage of providing spatially resolved results, thus giving $n_{e}, T_{e}$ and $T_{g}$ as a function of radial position. This opens the possibility to study the internal behavior of the discharge and the effect of the hydrogen impurities in detail. It is found that $\mathrm{H}_{2}$ introduction leads to plasma contraction, enhances the departure from equilibrium and leads to conditions that are close to those found in cool atmospheric plasmas. 
A disadvantage is that laser techniques are experimentally demanding and expensive. Therefore it is useful to perform passive and active spectroscopy quasi-simultaneously on the same plasma condition and position. By means of this poly-diagnostic calibration the validity region of the passive spectroscopic methods can be established so that future studies can eventually be based on passive methods only, for which a standard equipment for spectroscopic measurements and a calibration source suffices. When only passive methods are applied, it is expected that the changes in the spatial plasma structure as response to the introduction of molecules or power reduction can be obtained by analyzing pictures of the plasmas. This interesting topic is left for future studies.

The electron density has been measured under three different experimental conditions with three different methods, TS, Stark broadening, and ACI. A good agreement is found which proves that the passive method of ACI measurements is a suitable tool to measure $n_{e}$ in small atmospheric plasmas created on pure argon and also on argon-hydrogen mixtures. Nonetheless, the strong dependence of the ACI measurements on the gas temperature, electron temperature and plasma size implies that good approximations of these parameters are needed in order to obtain precise results.

For the case of electron temperature, the agreement found in the results obtained with TS and ALI on a pure argon plasma suggests that the ALI method provides a reliable option for the $T_{e}$ determination. However, when $\mathrm{H}_{2}$ is introduced in order to approach the CAP conditions, we see that the agreement between ALI and TS no longer holds.

\section{Acknowledgement}

This work, supported and financed by the Dutch Technology Foundation STW (project nr 6265) and the company Draka communications is performed in the framework of the bilateral relation between the Universities in Eindhoven (the Netherlands) and Cordoba (Spain). The Spanish Ministry of Education and Science is also acknowledged for partially supporting this research under the project No FIS2009-11191. The authors would like to thank Marco van de Sande and Toine Hendriks for their help with the setup and for their suggestions during fruitful discussions.

\section{References}

[1] Koinuma H, Ohkubo H, Hashimoto T, Inomata K, Shiraishi T, Miyanaga A and Hayashi S 1992 Appl. Phys. Lett. 60 816-817.

[2] Brok W J M, Bowden M D, van Dijk J, van der Mullen J J A M and Kroesen G M W 2005 J. Appl. Phys. 98013302

[3] Laroussi M and Lu X 2005 App. Phys. Lett. 87113902.

[4] de Regt J M, de Groote F P J, van der Mullen J J A M and Schram D C 1996 Spectrochimica Acta Part B $\mathbf{5 1} 13711383$

[5] de Vries N, Palomares J M, Iordanova E, van Veldhuizen E M and van der Mullen J J A M 2008 J. Phys. D: Appl. Phys. 41205203

[6] Moisan M, Sauvé G, Zakrewski Z and Hubert J 1994 Plasma Sources Sci. Technol. 3 584-592

[7] Jin Q, Zhu C, Borer M and Hieftje G M 1991 Spectrochim. Acta Part B 46 417-430

[8] Huang M, Hanselman D S, Jin Q and Hieftje G M 1990 Spectrochim. Acta B 45 1339-1352

[9] Bowden M D, Okamoto T, Kimura F, Muta H, Uchino K, Muraoka K, Sakoda T, Maeda M, Manabe Y, and Kimura T 1993 J. Appl. Phys. 732732

[10] Prokisch C, Bilgic A M, Voges E, Broekaert J A C, Jonkers J, van Sande M J and van der Mullen J J A M 1999 Spectrochim. Acta B. 54 1235-1266

[11] Narishige S, Suzuki S, Bowden M D, Uchino K, Muraoka K, Sakoda T and Park W Z 2000 Jpn. J. Appl. Phys. Part 1-Regular Pap. Short Notes Rev. Pap. $396732-6736$

[12] Warner K and Hieftje G M 2002 Spectrochim. Acta B. 57 201-241

[13] Jonkers J, de Regt J M, van der Mullen J J A M, Vos H P C, de Groote F P J and Timmermans E A H 1996 Spectrochim. Acta B. 51 1385-1392 
[14] van der Mullen J J A M, Boidin G and van de Sande M J 2004 Spectrochim. Acta Part B 59 929-940

[15] van der Mullen J J A M, van de Sande M J, de Vries N, Broks B, Iordanova E, Gamero A, Torres J and Sola A 2007 Spectrochimica Acta Part B 62 1135-1146

[16] Marshall K A and Hieftje G M 1987 J. Anal.Atom.Spectr osc. 2 567-571

[17] Muraoka K, Uchino K and Bowden M D 1998 Plasma Phys. Control. Fusion 40 1221-1239

[18] Jonkers J, Hartgers A, Selen L J M, van der Mullen J J A M and Schram D C 1999 Plasma Sources

Sci. Technol. 8 49-57

[19] Rousseau A, Teboul E, van de Sande M J and van der Mullen J J A M 2002 Plasma Sources Sci.

Technol. 11 47-52

[20] Gigosos M A and Cardenoso V 1996 J. Phys. B: At. Mol. Opt. Phys 29 4795-4838

[21] Gigosos M A, Gonzalez M A and Cardeñoso V 2003 Spectrochim. Acta Part B 58 1489-1504

[22] Iordanova E, de Vries N, Guillemier M and van der Mullen J J A M 2008 J. Phys. D: Appl. Phys. 41

015208

[23] de Vries N, Iordanova E, Hartgers A, van Veldhuizen E M, van den Donker M J and van der Mullen J

J A M 2006 J. Phys. D: Appl. Phys. 39 4194-4203

[24] Iordanova E, Palomares J M, Gamero A, Sola A and van der Mullen J J A M 2009 J. Phys. D: Appl. Phys. 42155208

[25] Moisan M, Zakrzewski Z and Pantel R 1979 J. Phys. D: Appl. Phys. 12 219-237

[26] de Vries N, Palomares J M, van Harskamp W J, Iordanova E, Kroesen G M W and van der Mullen J J

A M 2008 J. Phys. D: Appl. Phys. 41105209

[27] van de Sande M J and van der Mullen J J A M 2002 J. Phys. D: Appl. Phys. 35 1381-1391

[28] Palomares J M, Iordanova E, van Veldhuizen E M, Baede L, Gamero A, Sola A and van der Mullen J

J A M 2010 Spectrochim. Acta Part B doi:10.1016/j.sab.2010.03.001 (accepted for publication)

[29] Howard J, James B W and Smith W I B 1979 J. Phys. D: Appl. Phys. 12 1435-1440

[30] de Regt J M, de Groote F P J, van der Mullen J J A M and Schram D C 1996 Spectrochim. Acta B 51

$1527-1534$

[31] Yubero C, Calzada M D and Garcia M C 2005 J. Phys. Soc. Jpn. 74 2249-2254

[32] Shiu Y J and Biondi M A 1978 Phys. Rev. A. 17 868-872

[33] Schram DC, VanDerMullen JAM, DeRegt JM, Benoy DA, GEy GHAG, DeGrootte F and Jonkers J

1996 J. of Anal. At. Spectrom. 11 623-632

[34] Jonkers J, van de Sande M, Sola A, Gamero A, Rodero A and van der Mullen J J A M 2003 Plasma

Sources Sci. Technol. 12 464-474.

[35] Rolin M N, Shabunya S I, Rostaing J C and Perrin J M 2007 Plasma Sources Sci. Technol. 16480 491.

[36] Meulenbroeks R F G, Engeln R A H, Beurskens M N A, Paffen R M J, van de Sanden M C M, van der Mullen J J A M and Schram D C 1995 Plasma Sources Sci. Technol. 4 74-85

[37] Meulenbroeks R F G, Engeln R A H, Box C, de Bari I, van de Sanden M C M, van der Mullen J J A M and Schram D C 1995 Phys. Plasma 21002

[38] Beuthe T G and Chang J S 1999 Jpn. J. Appl. Phys. 38 4576-4580.

[39] Jonkers J, Selen L J M, van der Mullen J J A M, Timmermans E A H and Schram D C 1997 Plasma Sources Sci.\& Technol. 6 533-539.

[40] van der Mullen J J A M 1990 Phys. Rep. 191 109-220. 